\section{Available at \\ www.ComputerScienceWeb.com

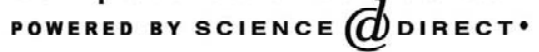

Medical Image Analysis 7 (2003) 251-264
$\overline{\overline{M E D I C A L}}$ IMAGE

ANALYSIS

www.elsevier.com/locate/media

\title{
Analyzing the neocortical fine-structure
}

\author{
F. Kruggel $^{\mathrm{a}, *}$, M.K. Brückner ${ }^{\mathrm{b}}$, Th. Arendt ${ }^{\mathrm{b}}$, C.J. Wiggins ${ }^{\mathrm{a}}$, D.Y. von Cramon ${ }^{\mathrm{a}}$ \\ ${ }^{\mathrm{a}}$ Max-Planck-Institute of Cognitive Neuroscience, Stephanstraße 1, 04103 Leipzig, Germany \\ ${ }^{\mathrm{b}}$ Paul-Flechsig-Institute for Brain Research, Jahnallee 59, 04109 Leipzig, Germany
}

Received 4 October 2001; received in revised form 3 December 2002; accepted 20 January 2003

\begin{abstract}
Cytoarchitectonic fields of the human neocortex are defined by characteristic variations in the composition of a general six-layer structure. It is commonly accepted that these fields correspond to functionally homogeneous entities. Diligent techniques were developed to characterize cytoarchitectonic fields by staining sections of post-mortem brains and subsequent statistical evaluation. Fields were found to show a considerable interindividual variability in extent and relation to macroscopic anatomical landmarks. With upcoming new high-resolution magnetic resonance imaging (MRI) protocols, it appears worthwhile to examine the feasibility of characterizing the neocortical fine-structure from anatomical MRI scans, thus, defining neocortical fields by in vivo techniques. A fixated brain hemisphere was scanned at a resolution of approximately $0.3 \mathrm{~mm}$. After correcting for intensity inhomogeneities in the dataset, the cortex boundaries (the white/grey matter and grey matter/background interfaces) were determined as a triangular mesh. Radial intensity profiles following the shortest path through the cortex were computed and characterized by a sparse set of features. A statistical similarity measure between features of different regions was defined, and served to define the extent of Brodmann's Areas 4, 17, 44 and 45 in this dataset.
\end{abstract}

(C) 2003 Elsevier B.V. All rights reserved.

\section{Introduction}

When discussing structure-function relationships in the brain, it is important to distinguish between anatomical and functional variability. While the latter is related to interindividual differences in the implementation of a cognitive task in the brain, task-solving strategies, motivation, etc., the former term addresses interindividual differences in the pattern, location and extent of functional activations. There is little doubt that there is a close correspondence between the functional organization of the neocortex and the cytoarchitectonic fields, which have been characterized by different histological staining techniques (e.g. Nissl staining) in post-mortem brains for about the last 100 years (Brodmann, 1908; Sakissov et al., 1955; Vogt and Vogt, 1919; von Economo, 1927). Cytoarchitectonic fields are defined by varying compositions of the general six-layered fine-structure of the neocortex characterized by the prop-

\footnotetext{
*Corresponding author. Tel.: +49-341-994-0223; fax: +49-341-9940221 .

E-mail address: kruggel@cns.mpg.de (F. Kruggel).
}

erties and densities of neurons and their connecting fibers (see Fig. 1). One of the most recent techniques for delineating the borders of cytoarchitectonic fields is called objective cytometry (Schleicher and Zilles, 1990; Schleicher et al., 1998). This technique examines radial intensity profiles across the neocortical sheet in stained brain sections, which are compared statistically along a trajectory on the surface. Local maxima in the classification function indicate a border between two fields.

It is now well accepted these fields show a considerable interindividual variability with respect to macroscopic landmarks such as sulcal and gyral lines and their substructures (Amunts et al., 1999; Rademacher et al., 1995; Rajkowska and Goldman-Rakic, 1995a,b; Weis et al., 1989). It is an open issue whether macroscopic landmarks are sufficient for describing the position of functional activation (e.g. as revealed by in vivo magnetic resonance (MR) scanning), or whether it is necessary to resort to atlas-based descriptions of cytoarchitectonic fields, that are obtained in vitro from different subjects in the form of a probabilistic map.

Myelin preparations of brain slices reveal the 'wiring' of 

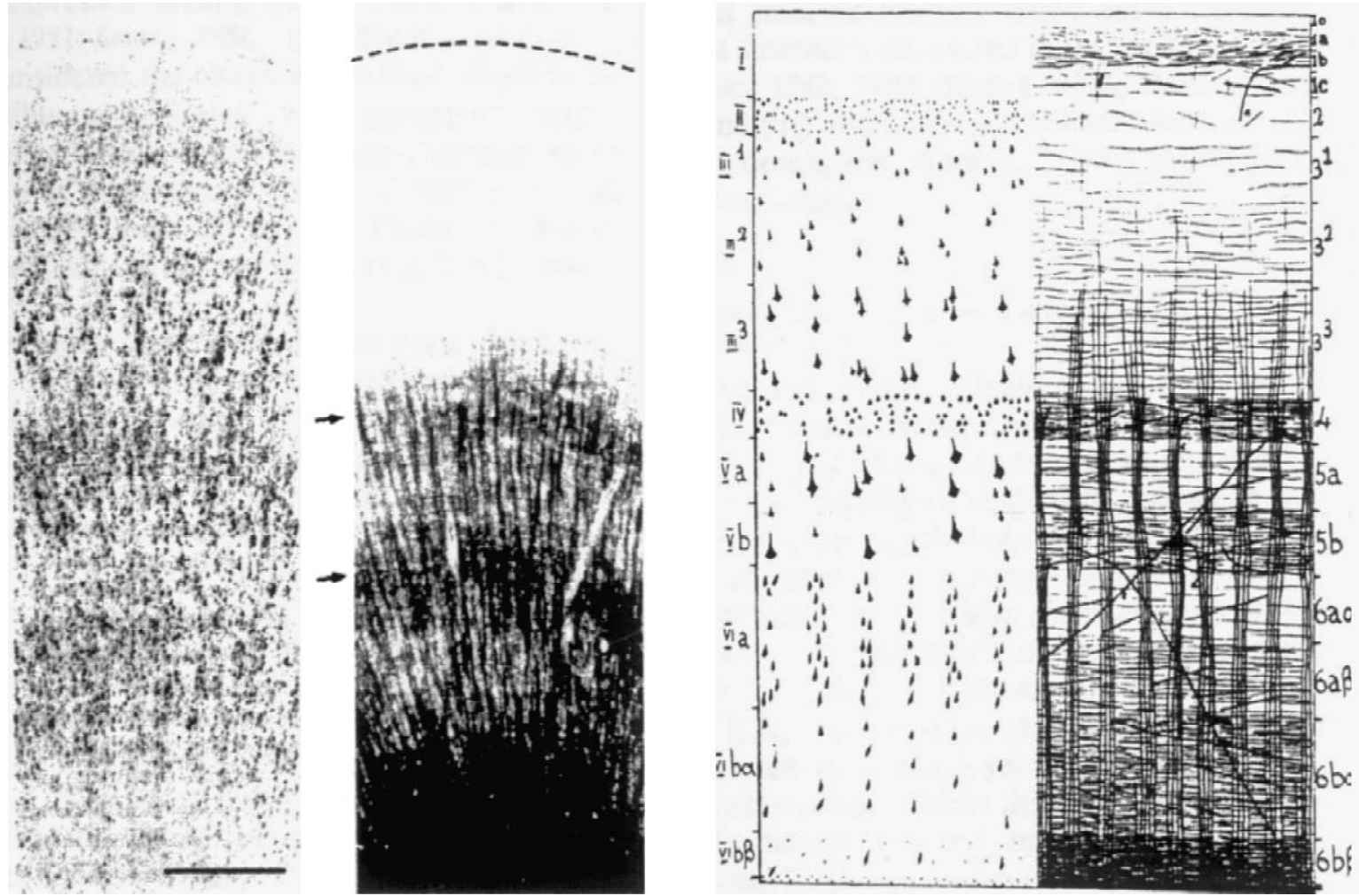

Fig. 1. Left: Nissl and myelin preparation from a section of Area 7 (reproduced from (Hellwig, 1993)). Right: Basic scheme of the cyto- and myeloarchitecture of an association cortex (reproduced from (Vogt and Vogt, 1919)). Note the similarity of the Myelin staining with the MR images of a fixated brain (e.g. see Fig. 3).

the neurons. There is some complimentarity between a myelin preparation and a Nissl staining (see Fig. 1). Neocortical subdivisions by cytoarchitecture resemble those based on myeloarchitecture.

Histological techniques offer a spatial resolution which is well beyond the limits of current (macroscopic) anatomical MR scanning protocols. However, recent investigations revealed that a spatial resolution of $0.25 \mathrm{~mm}$ (i.e. a matrix of $1024 \times 1024$ voxels) for anatomical scanning is feasible, and even $0.10 \mathrm{~mm}$ voxel dimensions may be aimed at for regional measurements. At this resolution, the neocortical sheet is mapped as a layer of 12 (or even, 30) voxels, which may be sufficient to recognize the layer structure of the cortex. Suitable image post-processing techniques may be designed to classify cortical intensity profiles, and thus, to define borders of cytoarchitectonic fields in vivo.

The work described here investigates the feasibility of segmenting the neocortical fine-structure in MR data. To achieve a high spatial resolution at a reasonable signal-tonoise ratio (SNR) without restrictions in scanning time, we have resorted to examine a fixated brain hemisphere. Since the MR signal strength is related to the local cellular environment (e.g. biopolymer content) in a volume, it is not unreasonable to assume that stained histological intensity profiles and MR intensity profiles show some similarity, albeit at a much lower spatial resolution. Thus, we will compare previously published results obtained by objective cytometry (Amunts et al., 1995, 1997, 1999; Geyer et al., 1999; Zilles et al., 1986) with a MR-based neocortical fine-structure analysis.

\section{Materials and methods}

Most of the image processing techniques applied here are well described in the literature. However, some details were changed with respect to the original implementation. In order to enhance the reproducibility of the work presented here, we have chosen to include a detailed description of our analysis algorithms.

\subsection{Brain preparation and scanning}

An isolated left brain hemisphere (female, 72 years of age) was obtained from an routine autopsy. The time between death and extraction of the hemisphere was less than $24 \mathrm{~h}$. The specimen was fixed in formalin and embedded in a small container in agar gel, taking care of removing small air bubbles located in deep sulci by ultrasound immersion. MR acquisition was performed on a Bruker 3 T Medspec 100 system equipped with a bird cage quadrature coil using a $T_{1}$-weighted 3D MDEFT protocol (Lee et al., 1995), FOV $96 \times 192 \times 128 \mathrm{~mm}$, matrix $256 \times$ 
$512 \times 512$, voxel size $0.375 \times 0.375 \times 0.25 \mathrm{~mm}$, scanning time $12 \mathrm{~h}$. Other than the number of acquisitions and the field-of-view, parameters of our anatomical MR imaging protocol remained at their standard setting. Fig. 2(top) shows example views from this dataset.

\subsection{Preprocessing}

Scan data were interpolated to an isotropical voxel size of $0.25 \mathrm{~mm}$ by a fourth-order b-spline method (Thevenaz et al., 2000) and cropped to a minimum bounding box enclosing the hemisphere of $276 \times 608 \times 384$ voxels.

Intensity inhomogeneities were corrected by a modification of the adaptive fuzzy clustering algorithm (AFCM, Pham and Prince, 1999). The intensity variation in the image $y$ is modeled by multiplying the centroids $c_{k}$ by some unknown multiplier field $m$ which is assumed to be smooth and slowly varying. The AFCM algorithm seeks to minimize the cost function:

$$
\begin{aligned}
J_{\mathrm{AFCM}}= & \sum_{j \in \Omega} \sum_{k=1}^{K} u_{j k}^{2}\left(y_{j}-m_{j} c_{k}\right)^{2}+\lambda_{1} \sum_{j \in \Omega} \sum_{a=1}^{3}\left(D_{a} \star m\right)_{j}^{2} \\
& +\lambda_{2} \sum_{j \in \Omega} \sum_{a=1}^{3} \sum_{b=1}^{3}\left(D_{a} \star D_{b} \star m\right)_{j}^{2},
\end{aligned}
$$

where $u_{j k}$ denote the class membership probabilities, $D_{a}$ and $D_{b}$ the finite difference operators along directions $a$ and $b, \star$ the convolution operator, and $\lambda_{1}, \lambda_{2}$ correspond to Langrange multipliers. The steps of our variant of the AFCM algorithm can be described as follows:

1. For initializing the multiplier field, we assume that the mean intensity of the foreground (tissue) voxels within a certain cubical window is constant. The image is divided into cubical regions $w$ of size $s$. The mean
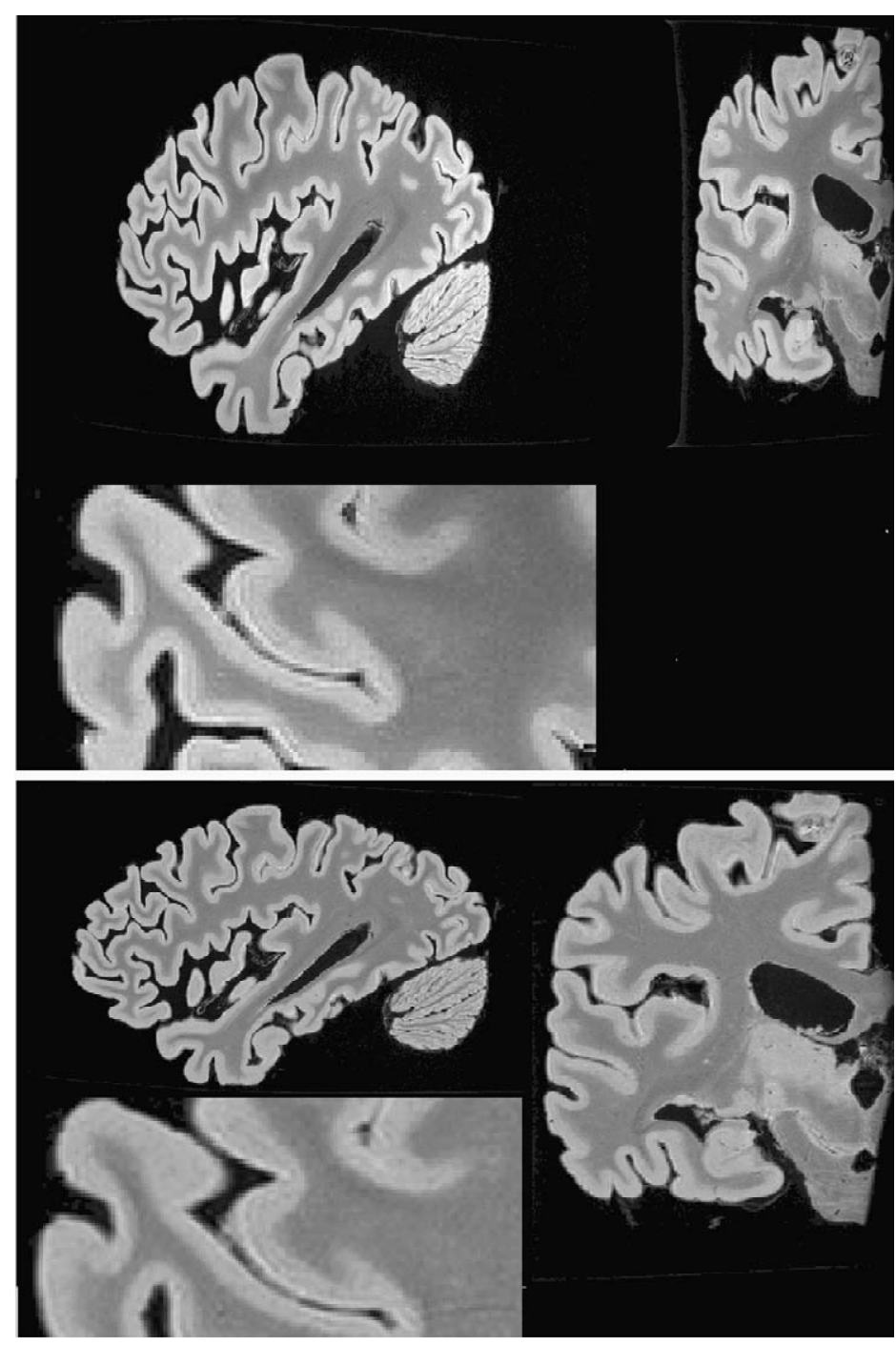

Fig. 2. Top: Sagittal, coronal and zoomed-in axial view of the original high-resolution MRI dataset. Note the anisotropic voxel size and the intensity inhomogeneities, especially close to the midline structures and in the cranio-caudal direction. Below: Corresponding views of the interpolated and intensity-corrected dataset. Note that the $T_{1}$ contrast is 'inverted' by fixation: regions of higher neuron content (i.e. cortex, basal ganglia) show a higher signal intensity than fiber-containing regions (i.e. the white matter). 
intensity $\bar{y}_{w}$ of all voxels above an intensity threshold lim within a window is computed. The initial value for the multiplier field within this region is set to $m_{w}=\bar{y}$ / $\bar{y}_{w}$, where $\bar{y}$ denotes the mean intensity of the whole image. This multiplier field is expanded up to the original image resolution using trilinear interpolation. We used $s=16$ and $\lim =35$ for our dataset. Initial values for the class centroids $c_{k}$ are obtained by a simple $k$-means clustering, respecting the local value of the multiplier field.

2. The class probability memberships are computed by

$$
u_{j k}=\frac{\left(y_{j}-m_{j} c_{k}\right)^{-2}}{\sum_{k=1}^{K}\left(y_{j}-m_{j} c_{k}\right)^{-2}} \quad \forall j \in \Omega \text { and } k=1, \ldots, K
$$

3. New values for the centroids are obtained from

$$
c_{k}=\frac{\sum_{j \in \Omega} u_{j k}^{2}\left(y_{j}-m_{j} y_{j}\right)}{\sum_{j \in \Omega} u_{j k}^{2} m_{j}} \quad \forall k=1, \ldots, K .
$$

4. Finally, a new multiplier field is determined by solving the equation

$y_{j} \sum_{k=1}^{K} u_{j k}^{2} c_{k}=m_{j} \sum_{k=1}^{K} u_{j k}^{2} c_{k}^{2}+\lambda_{1}\left(m_{j} \star H_{1}\right)+\lambda_{2}\left(m_{j} \star H_{2}\right)$.

Refer to (Pham and Prince, 1999) for a definition of the convolution masks $H_{1}$ and $H_{2}$. This equation is solved using successive overrelaxation. We used $\lambda_{1}=20000$ and $\lambda_{2}=200000$.

5. Steps $2-5$ are repeated until the algorithm has converged. Typically, the maximum change in the membership values between iterations is less than 0.01 after 20 iterations.

This algorithm was applied to yield a segmentation into three classes (background: BG, grey matter: GM, white matter: WM) and an intensity-corrected version of the input image (see Fig. 2(below)).

The cerebellum and brainstem were manually removed in the WM segmentation at the level of the superior colliculus using an image editor (Kruggel and Lohmann,
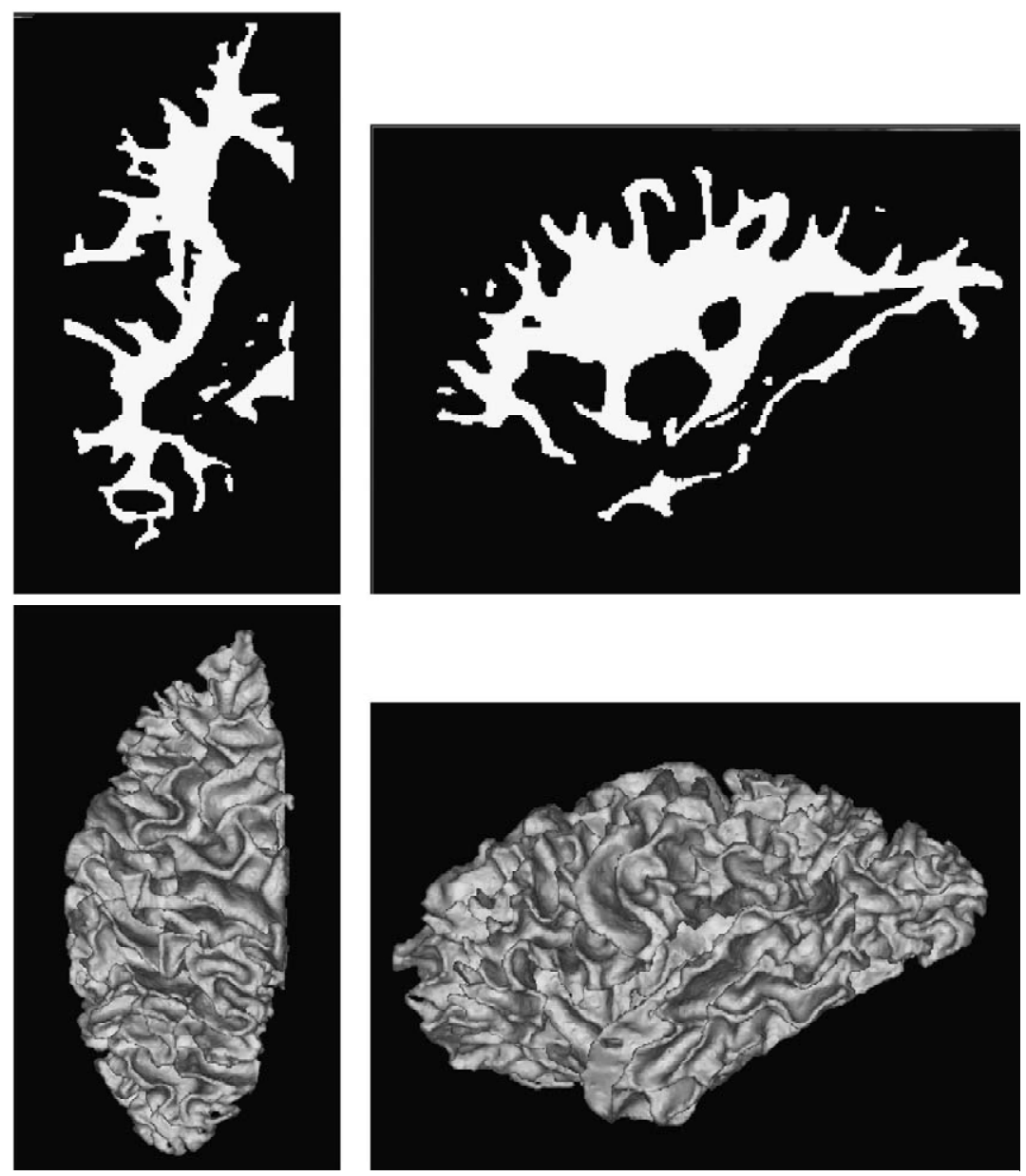

Fig. 3. Top: Axial (left) and sagittal (right) view of the WM segmentation. Below: Corresponding views of the initial WM surface mesh (200k vertices). 

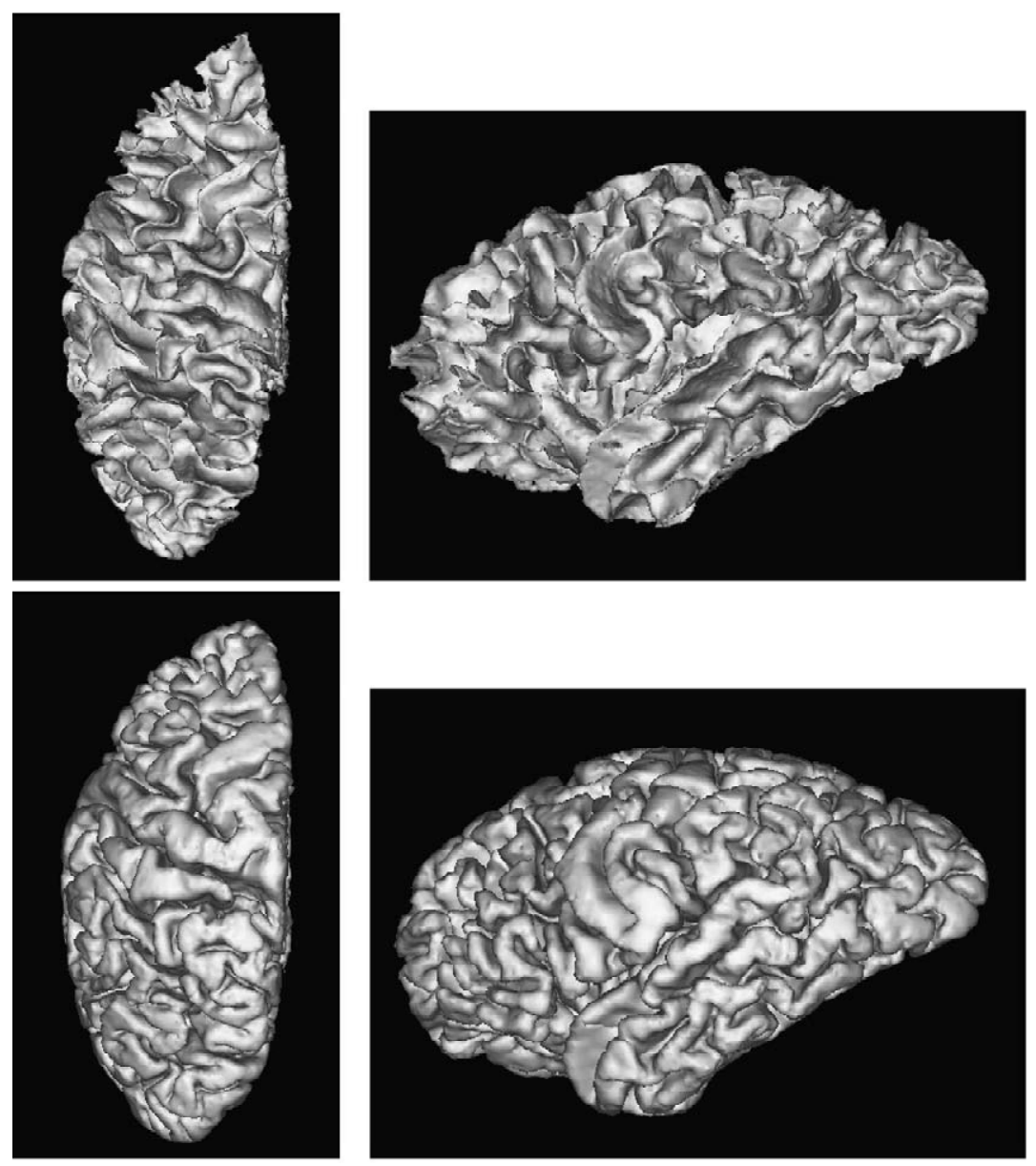

Fig. 4. Top: Axial (left) and sagittal (right) view of the adapted WMS mesh. Below: Corresponding views of the initial GMS mesh.

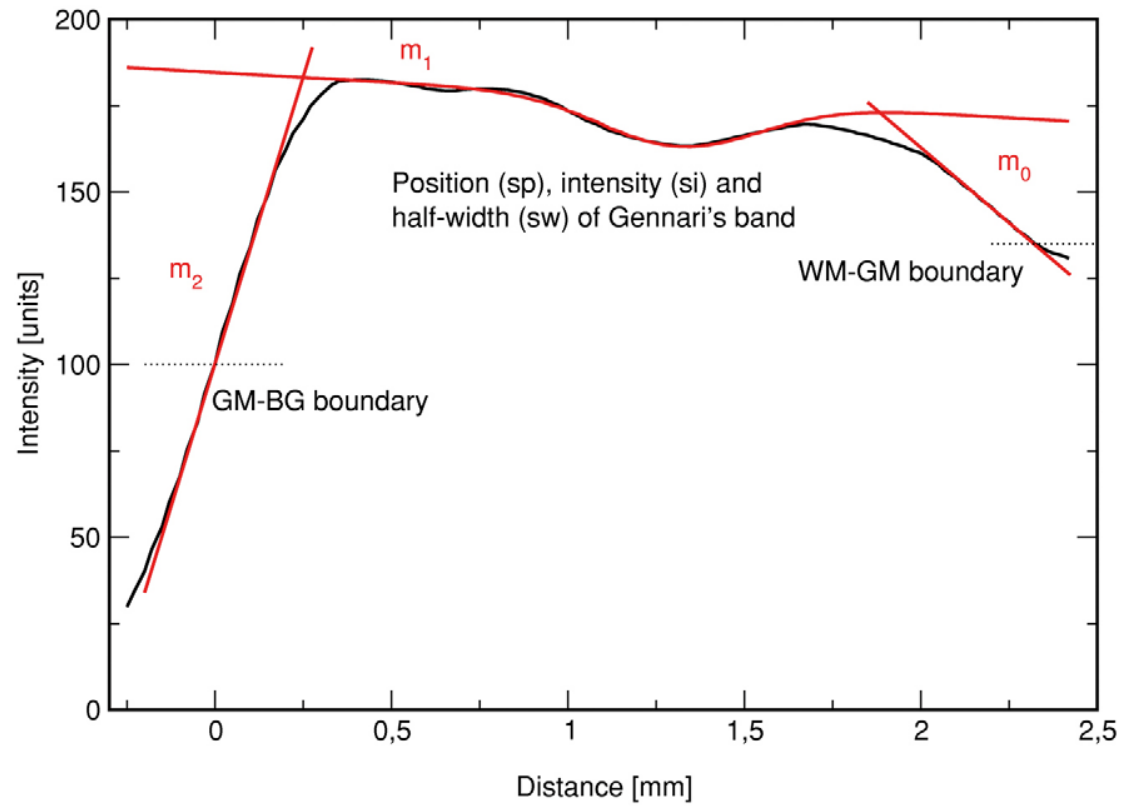

Fig. 5. Example intensity profile across Area 17. The rising flank (on the left) crosses the WM-GM border, whereas the slope of the intra-cortical segment is comparatively flat. The falling flank mostly results from the partial volume effect on the GM-BG boundary. A Gaussian function is used to model the position, intensity and width of intra-cortical bands, such as Gennari's band. 
1996) and smoothed by a rank filter to yield a voxel-based representation of the cerebral WM compartment.

\subsection{Surface generation}

A raw triangular surface mesh was generated from the WM segmentation using the marching tetrahedra algorithm (Payne and Toga, 1990) and decimating the initial mesh from 1.9 Mio to $200 \mathrm{k}$ faces while respecting the local curvature by a quadrics-based algorithm (Garland and
Heckbert, 1999). Refer to Fig. 3 for an example view of the WM segmentation and the initial mesh.

An improved model of the WM surface (WMS) was obtained by treating this initial WM surface as a deformable model (Dale and Sereno, 1993; Xu and Prince, 1998; Kruggel and von Cramon, 2000).

On any vertex $\vec{v}_{0}$ in the mesh, internal and external forces act until a balance is achieved. The internal force $\overrightarrow{\boldsymbol{F}}_{\text {int }}$ tries to center a vertex among its edge-connected neighbors $\left\{\vec{v}_{i}\right\}_{i=1, N}$ :
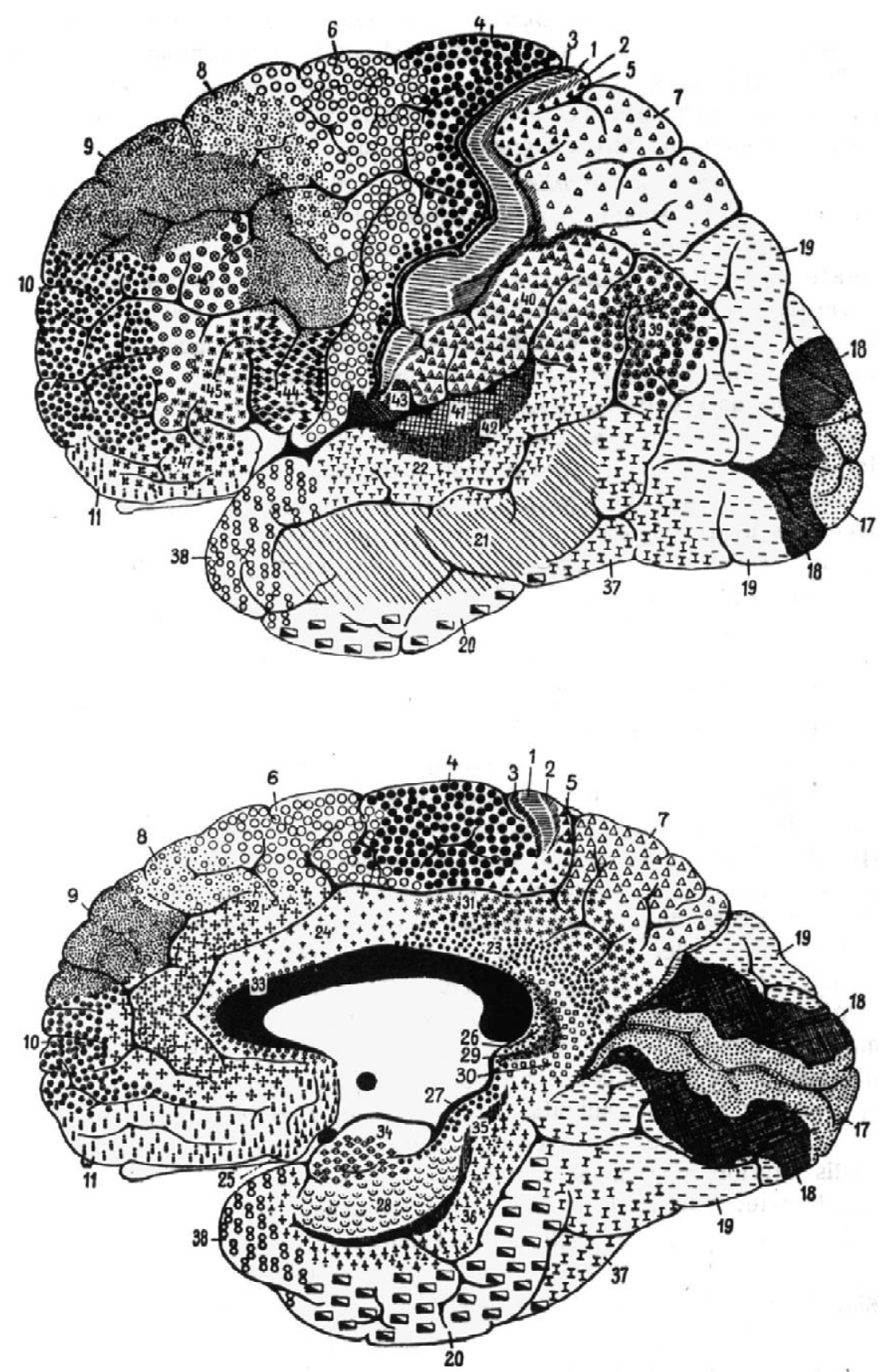

Fig. 6. Map of the cytoarchitectonic fields according to Brodmann (reproduced from (Brodmann, 1908)). 
$\overrightarrow{\boldsymbol{F}}_{\mathrm{int}}=\frac{1}{N} \sum_{i=1}^{N}\left(\overrightarrow{\boldsymbol{v}}_{i}-\overrightarrow{\boldsymbol{v}}_{0}\right)$

The first external force $\overrightarrow{\boldsymbol{F}}_{\text {ext, } 1}$ tears a vertex outwards along the direction of its surface normal $\overrightarrow{\boldsymbol{n}}_{0}$. This force is exerted by an intensity-gradient field $f$, that is computed from a convolution of the intensity-corrected image $I$ with a Gaussian kernel $G$ : $f=\nabla(G \star I)$ (Xu and Prince, 1998):

$\left|\overrightarrow{\boldsymbol{F}}_{\mathrm{ext}, 1}\right|=f\left(\overrightarrow{\boldsymbol{v}}_{0}\right) \bullet \overrightarrow{\boldsymbol{n}}_{0}$,

where $\rightarrow$ denotes the inner product. The second external force $\overrightarrow{\boldsymbol{F}}_{\text {ext,2 }}$ captures the surface within a narrow range around an image intensity $I_{\text {lim }}$ :

$$
\left|\overrightarrow{\boldsymbol{F}}_{\mathrm{ext}, 2}\right|=\tanh \left(\kappa\left(I\left(\overrightarrow{\boldsymbol{v}}_{0}\right)-I_{\mathrm{lim}}\right)\right),
$$

where $\kappa$ corresponds to the capturing range and is related to the noise level in the data set (here, $\kappa=3$ ). $\overrightarrow{\boldsymbol{F}}_{\text {ext,2 }}$ pushes a vertex outside until a position with intensity $I_{\text {lim }}$ is reached, and inside if the intensity is too low. A suitable value for $I_{\text {lim }}$ was chosen as the mean intensity of the WM and GM class (here, $I_{\text {lim }}=135$ ). Forces are weighted to ensure good convergence properties during iterations $t$ of the surface adaption process:

$$
\begin{aligned}
\overrightarrow{\boldsymbol{v}}_{0}(t+1)= & \overrightarrow{\boldsymbol{v}}_{0}(t)+w_{1} \overrightarrow{\boldsymbol{F}}_{\mathrm{int}} \\
& +\overrightarrow{\boldsymbol{n}}_{0}\left(w_{2}\left|\overrightarrow{\boldsymbol{F}}_{\mathrm{ext}, 1}\right|\left|\overrightarrow{\boldsymbol{F}}_{\mathrm{ext}, 2}\right|+w_{3}\left|\overrightarrow{\boldsymbol{F}}_{\mathrm{ext}, 2}\right|\right)
\end{aligned}
$$
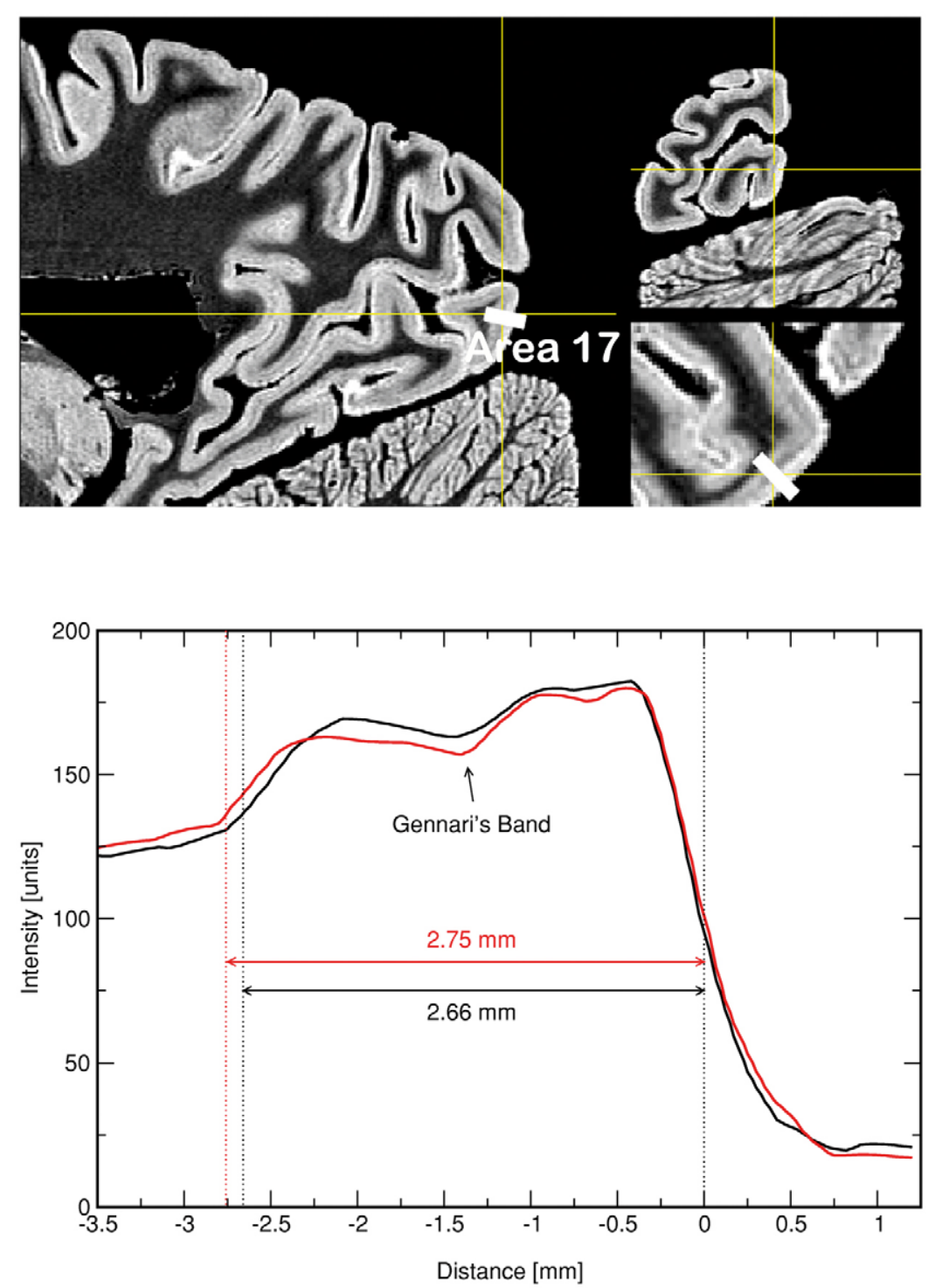

Fig. 7. Top: Sagittal, coronal and axial slice (enlarged) through the visual cortex. The position of the intensity profiles through Area 17 is marked by a white bar. Below: Two intensity profiles through Area 17. Gennari's band, which is visible as a dark horizontal line in the cortex, is indicated by an intensity drop at $\approx 52 \%$ of the cortical width. 
The image gradient force $\left|\vec{F}_{\text {ext, },}\right|$ is weighted by the intensity force $\left|\vec{F}_{\text {ext, }, 2}\right|$ to reduce the outward-driving force adaptively when the destination intensity range is reached. This constraint also reduces the chance of introducing self-intersections. The process is iterated until the sum of vertex position shifts falls below a pre-defined limit. The result is kept as the final WMS representation. For the WM surface, we used $w_{1}=0.01, w_{2}=0.02, w_{3}=0.02$. Similarly, the GM surface (GMS) was computed from the initial surface by setting $I_{\lim }=200, w_{1}=0.1, w_{2}=0.02, w_{3}=$ 0.02 . Refer to Fig. 4 for an example view of the refined WMS and GMS.

\subsection{Intensity profiles}

The next step was to compute intensity profiles across the cortex. For each vertex on the WMS, the closest point on the GMS was computed (Kruggel and von Cramon, 2000).

For a vertex $\vec{v}_{\mathrm{GMS}}$ of the GM surface mesh, the shortest distance to all triangles on the WMS was determined (Eberly, 1999). To avoid a brute force search, we implemented a 'spatial cache': for each voxel of the volume, we compiled a list of triangles touching this voxel. Since WMS and GMS originated from the same initial surface, a first guess for the closest WMS triangle is the one with the same mesh index. We limited the search for the closest triangle to a small subregion around this WMS triangle, guided by the spatial cache. The closest point $\vec{v}_{\text {WMS }}$ on the WMS was stored for each vertex $\vec{v}_{\mathrm{GMS}}$.
Along a line through both points, an intensity profile was sampled from the intensity-corrected image at regular intervals of $0.1 \mathrm{~mm}$. In order to define the WM-GM and GM-BG boundary points consistently, lines were adapted to the rising flank of the profile (at the GM-WM boundary) and to the falling flank (corresponding to the GM-BG boundary, see Fig. 5). The exact position of the GM-WM boundary was computed at intensity $I=135$ (corresponding to the mean of the intensities of the WM and GM class), for the GM-BG boundary at intensity $I=100$ (corresponding to the mean of the intensities of the GM and BG class), and their distance was recorded as the local cortical thickness th. Because layers occupy a rather constant relative portion of the cortex, the profile was resampled at $1 \%$ intervals of th between both boundaries. In summary, we obtained for each vertex on the WMS the cortical thickness and a normalized intensity profile of 101 data points.

\subsection{Modeling profiles}

Intensity profiles were characterized for statistical evaluation by (i) the slope of the rising flank at the GM-WM boundary $m_{0}$ (see Fig. 5), (ii) the slope of the intra-cortical portion $m_{1}$, (iii) the slope of the falling flank at the GM-BG boundary $m_{2}$. In addition, the position ( $(b p)$, intensity $(b i)$ and width $(b w)$ of an intra-cortical band were determined by adaptation of a Gaussian function to the intra-cortical profile segment using the model function $I=m_{1} * x+n_{1}-b i * \exp \left(-(x-b p)^{2} / b w^{2}\right) . \quad$ Powell's algorithm was used to find optimized parameters.

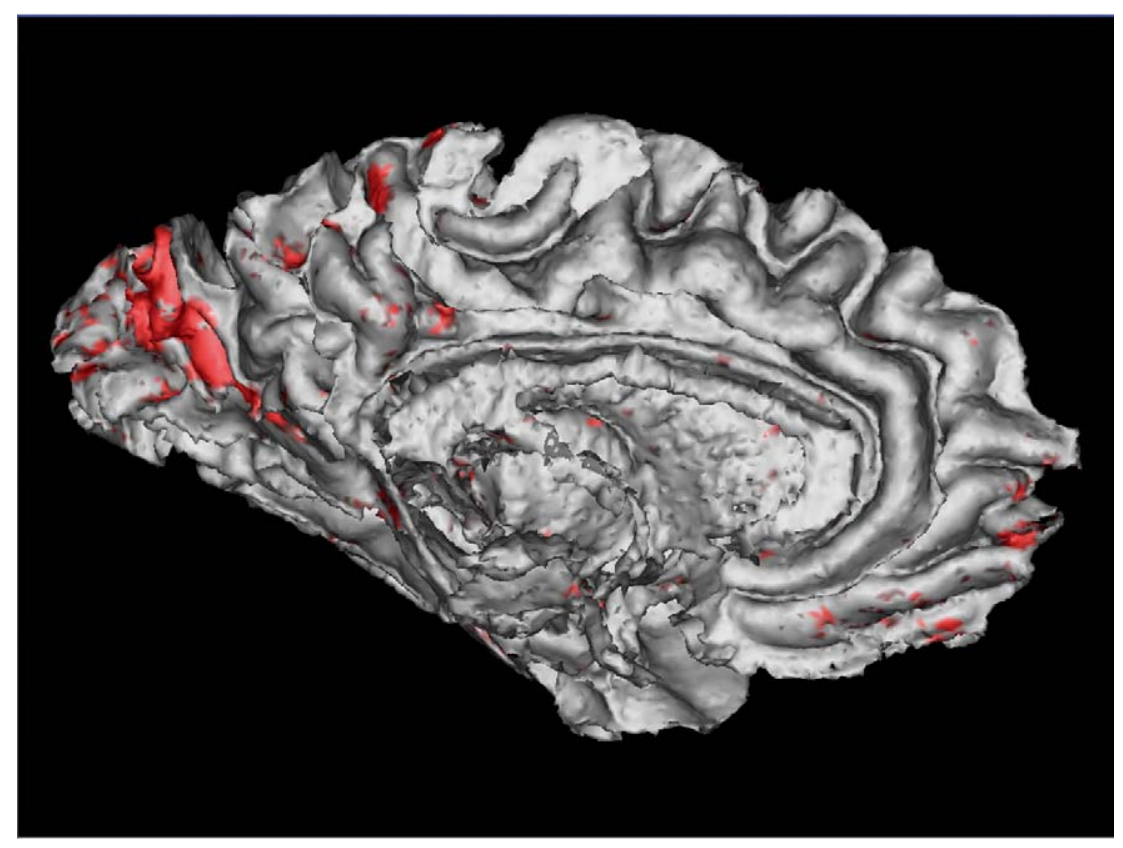

Fig. 8. Medial view of the white matter surface. The model region marked in Fig. 7 was used to detect area 17 which is mapped in color-code at a detection threshold of $z \geqslant-1$. 


\subsection{Statistical evaluation}

Cortical areas with similar fine structure were determined by comparing of local profile properties with those of a template region. To characterize the template, profile properties were collected from a surface patch of $5 \mathrm{~mm}$ diameter around a manually specified position (typically, 60-100 vertices). Properties of a local sample were collected from a given vertex and its first and second-order neighbors (typically, 10-30 vertices). From a series of heuristical experiments, six statistical tests were selected to measure the similarity of the local region with the template: $\left(z_{1}\right)$ Pearson's correlation coefficient of the averaged profile in both regions, $\left(z_{2}\right)$ Pearson's correlation coefficient of the first derivative of the averaged profiles, $\left(z_{3}\right)$ a $t$-test comparing the cortical thickness $t h$ in both areas, $\left(z_{4}\right)$ a $t$-test comparing the rising slope $m_{0}$ in both areas, $\left(z_{5}\right)$ a $t$-test comparing the intra-cortical slope $m_{1}$ in both areas, and $\left(z_{6}\right)$ a $t$-test comparing the band intensity $b i$ in both areas. $P$-values obtained from the tests were converted into $z$-scores, and a similarity measure was derived as: $z_{\text {sim }}=$ $z_{1}+z_{2}-\left|z_{3}\right|-\left|z_{4}\right|-\left|z_{5}\right|-\left|z_{6}\right|$. If both regions contain similar profiles, $z_{3}$ to $z_{6}$ contribute values close to 0 , while $z_{1}$ and $z_{2}$ provide positive scores, summing up to some (small) positive quantity. For dissimilar regions, negative similarity measures are expected. A threshold of $z_{\text {sim }} \geqslant$ -1 was used in all subsequent figures.

\section{Results}

We selected three different anatomical regions which are well studied by histological techniques. We were interested in comparing intensity profiles with the known descriptions of the local layer structure, and in comparing the extent of statistically homogeneous regions with known cyto-
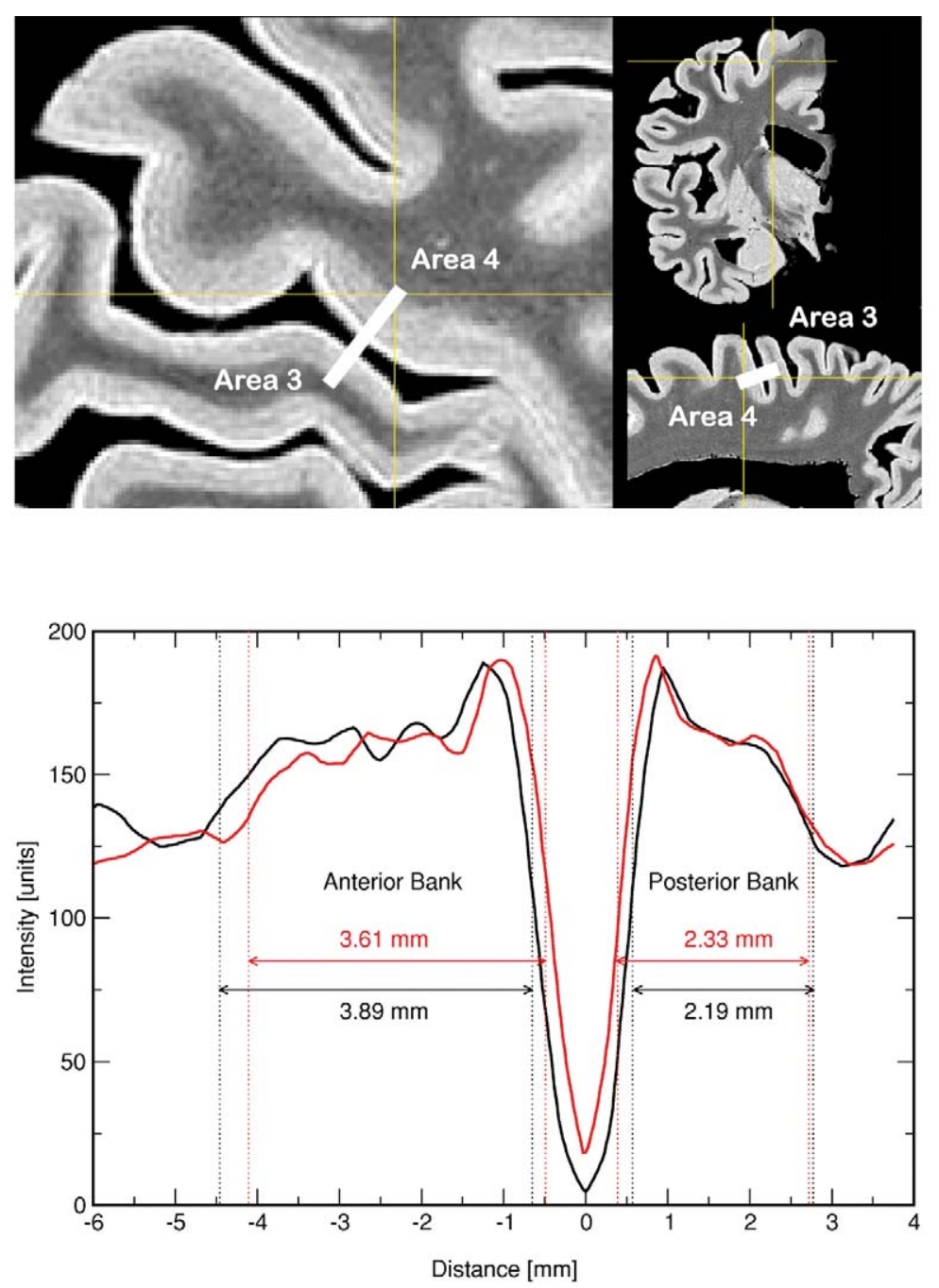

Fig. 9. Top: Sagittal, coronal and axial slice (enlarged) through the anterior (Area 4, motor cortex) and posterior (Area 3, sensory cortex) bank of the central sulcus. The position of the intensity profiles through Areas 4 and 3 is marked by a white bar. Below: Two intensity profiles through Area $4+3$. The motor cortex is thicker than the sensory cortex and shows more substructure. 
architectonic fields. Seminal work about the description of these fields was provided by Brodmann (1908), thus we refer to his classification as Brodmann's Areas (see Fig. 6).

Note that the $T_{1}$ contrast is 'inverted' by fixation: regions of higher neuron content (i.e. cortical layers 1-3, 5 and 6, basal ganglia) show a higher signal intensity than fiber-containing regions (i.e. the white matter).

\subsection{Visual cortex (Area 17)}

The visual cortex is distinguishable from the surrounding Area 18 by the presence of Gennari's band, which corresponds to layer $\mathrm{IVb}$ of this cortex and contains an intracortical horizontal fiber system. This structure is easily detected in the acquired MR dataset as a darker band in the bright cortex (see Fig. 7).

By application of the procedures described above, the cortical thickness on the banks of the calcarine fissure were determined as 1.86 $\pm 0.10 \mathrm{~mm}$ (von Economo, 1927: 1.84 $\mathrm{mm})$, the position of the center of Gennari's band as 52 $\pm 6 \%$ (Zilles et al., 1986: 55\%), and the thickness of this band as $0.30 \pm 0.10 \mathrm{~mm}$ (Zilles et al., 1986; $0.28 \mathrm{~mm}$ ).

The extent of Area 17 is described by von Economo (1927) as located on the walls and lips of the calcarine fissure, and at the gyral crowns at the occipital pole. This description compares nicely with the automatically generated statistical classification as shown in Fig. 8.

\subsection{Motor and sensory cortex (Area 4 and 3)}

As a second example, we tried to differentiate the primary motor cortex (Area 4) on the anterior bank of the central sulcus from the somatosensory cortex (Area 3) on its posterior bank (see Fig. 9).

The most distinctive feature here is the cortical thickness: on the anterior bank, the motor cortex reaches values up to $3.8 \mathrm{~mm}$, while the sensory cortex is less than $2.2 \mathrm{~mm}$ thick (von Economo, 1927; MacDonald et al., 2000). Intensity profiles in Area 4 mostly showed three maxima (see Fig. 9), which roughly correspond to the transition between layer II/III, layer III/V and layer V/VI as described by Amunts et al. (1995). The somatosensory cortex on the posterior bank exhibited much less substructure.

A statistical classification was initialized by a manually specified region close to the hand field and yielded the full extent of the motor cortex well in agreement with previously published histological classifications (see Fig. 10). The border between the anterior and the posterior bank is sharp, although some small spots especially at crowns of other gyri respond to this classificator as well.

\subsection{Broca's Area (Area 44 and 45)}

As a final example, we selected Broca's speech region, which corresponds to Area 44 (the pars opercularis of the inferior frontal gyrus) and Area 45 (the pars triangularis of the inferior frontal gyrus).

As described by von Economo (1927) and Amunts et al. (1999), the cortex of Area 44 is not sharply delineable from the white matter, which corresponds to a flat slope of $m_{0}$ (see Fig. 11, bottom right). The cortex of Area 45 (see Fig. 11, bottom left) is thinner and features a more distinct horizontal layering.

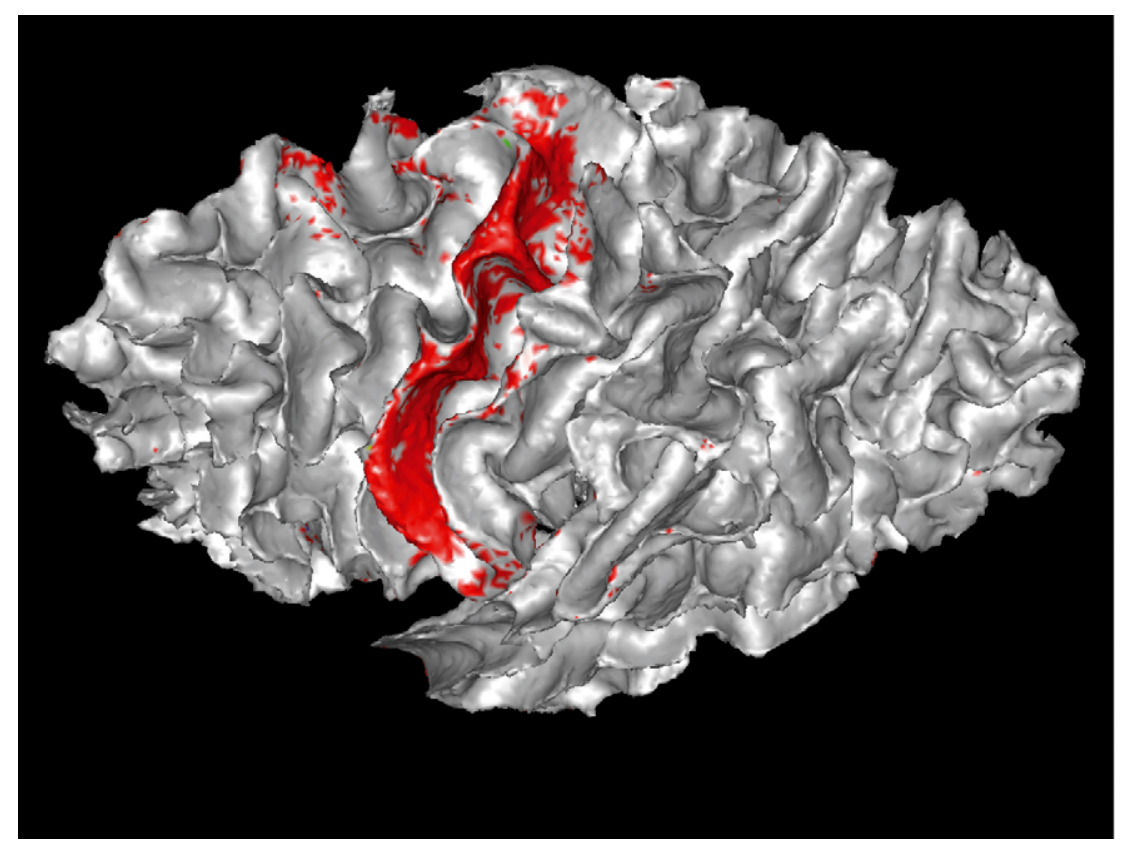

Fig. 10. Top lateral view of the white matter surface. Area 4 (detected from a model region on the anterior bank of the central sulcus, see in Fig. 9) is color-coded at a detection threshold of $z \geqslant-1$. 

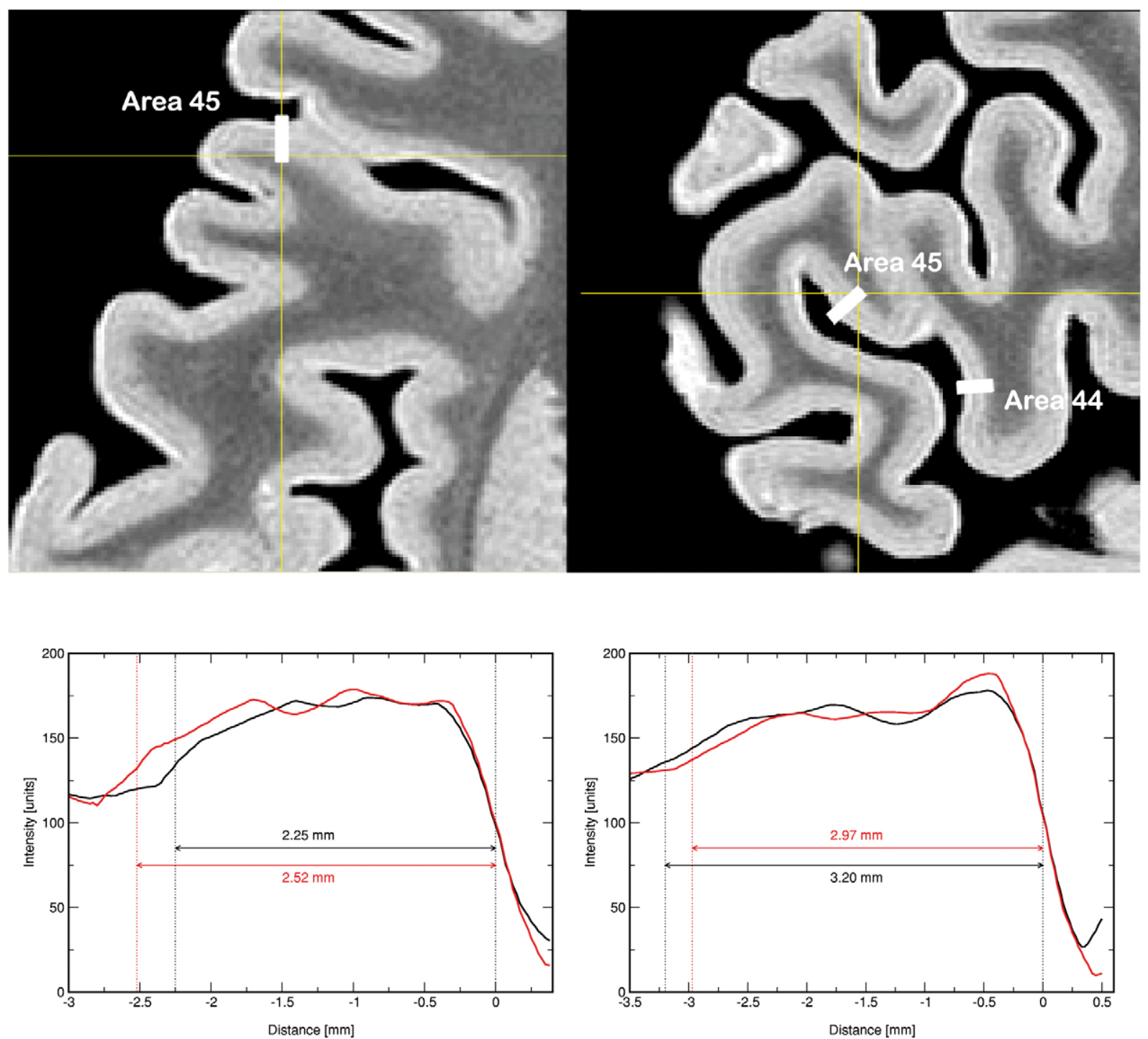

Fig. 11. Top: Axial (enlarged) and sagittal section through the inferior frontal gyrus. The profile denoted Area 45 was taken from the pars triangularis, while the other profile (Area 44) corresponds to the pars opercularis. Below: Intensity profiles through Area 45 (left) and Area 44 (right). The cortex is thinner in Area 45, but exhibits a more prominent banded structure.
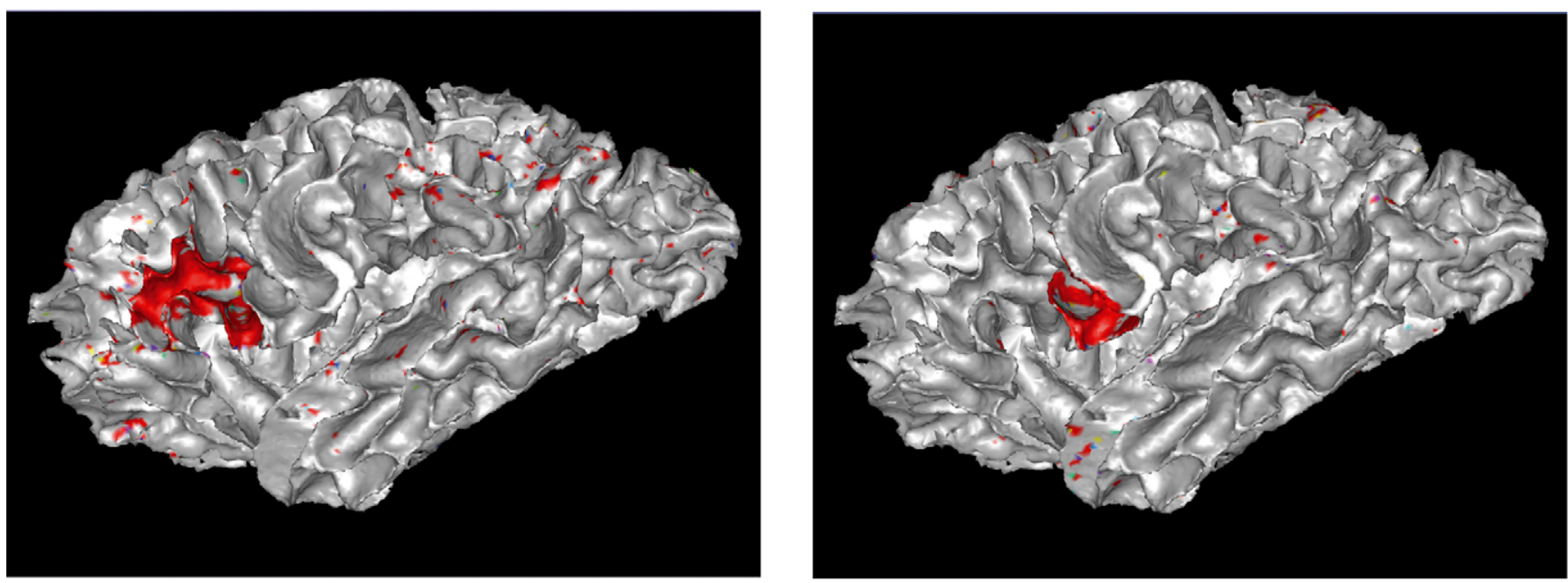

Fig. 12. Lateral view of the white matter surface. Area 45 (left) and Area 44 (right) were detected from model position shown in Fig. 11 at a detection threshold of $z \geqslant-1$. 
For the delineation the extent of both areas, training regions were selected at characteristic positions as indicated in Fig. 11. Classification results are shown superimposed on the white matter surface in Fig. 12, separated for Area 45 (top) and Area 44 (below).

\section{Discussion}

Results shown for three different brain areas demonstrate the feasibility of analyzing the neocortical substructure from high-resolution MRI data. The qualitative properties of the MRI intensity profiles and quantitative descriptors (e.g. cortical thickness, band position and width) corresponded well with descriptions found in reference publications based on histological examinations. Using statistical descriptors of the profiles obtained from a template region, the extent of target regions was de- termined by comparing local descriptors with the template. Regions found correspond well with prior knowledge from histological examinations.

We want to emphasize the preliminary nature of this feasibility study. A validation of our regional classification by histological examination of the same specimen is missing. Currently, we conduct an extensive study to compare results from high-resolution MRI scanning and histological staining in a group of fixated brains from healthy subjects and patients suffering from neurodegenerative diseases. Fig. 13 shows a comparison of an MR slice and a histological slice. After MR scanning, this slice was cut from the same hemisphere, Weigert-stained, embedded, digitized using a PC scanner and registered with the MR image volume. Note the striking qualitative similarity between the MR signal intensity and the contrast induced by staining. The correlation coefficient of the example intensity profile is 0.92 . Recently, similar results
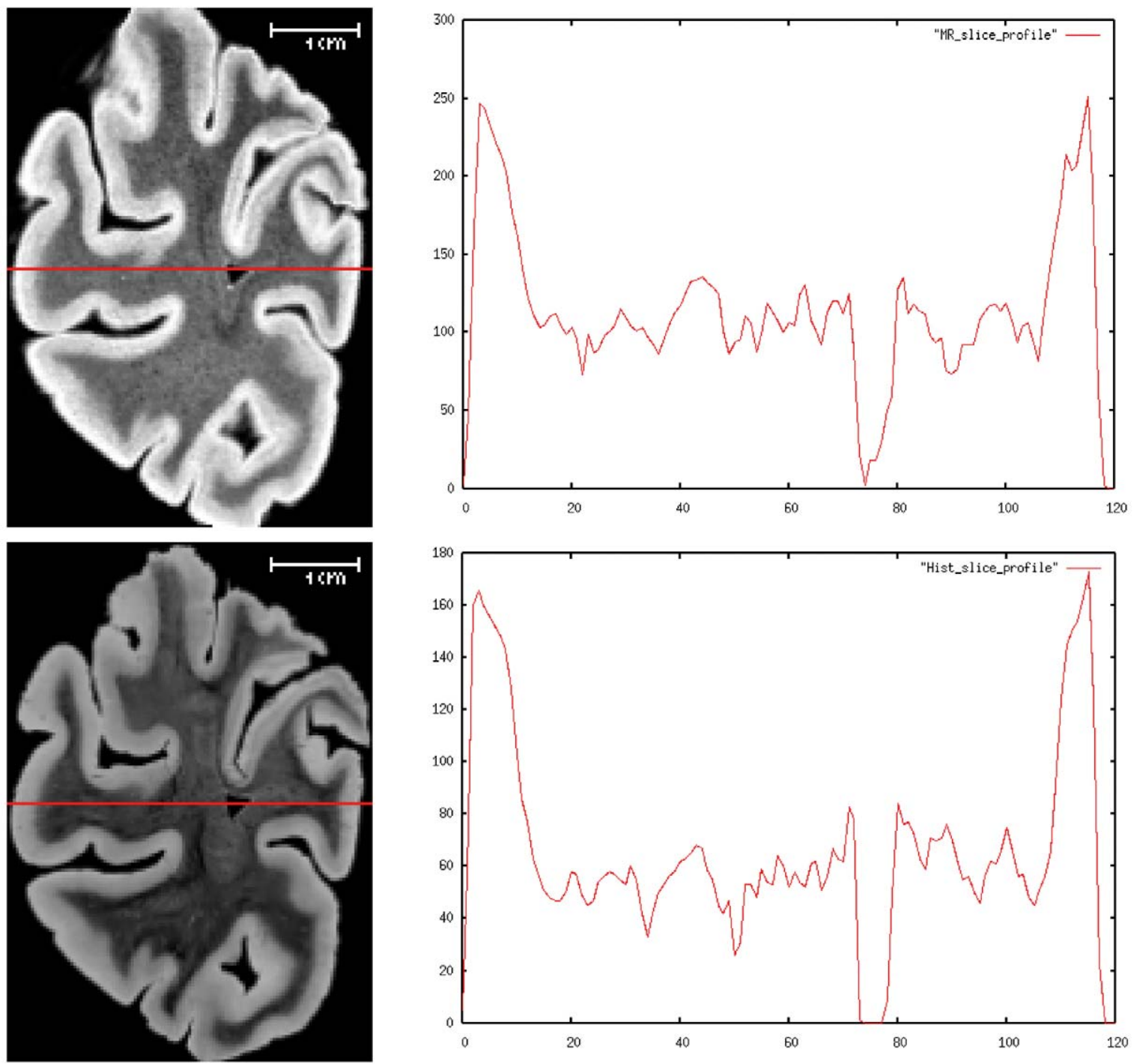

Fig. 13. Coronal section through the occipital lobe. MR slice (top) and registered Weigert-stained histological slice (below). Sample intensity profiles along the straight lines in both slices are shown on the right. The correlation coefficient for both profiles is 0.92 . 
were reported independently (Fatterpekar et al., 2002). Small brain excisions were scanned in an MR microscope (MRM) at 9.4 Tesla field strength at a resolution of $78 \times 78 \times 500 \mu \mathrm{m}$. Although a different acquisition protocol was used, this group also noted a good correspondence between stained slices and MRM images.

It is unclear how the well-known cortical cellular and fiber structure translates into intensity profiles as revealed by high-resolution MRI in fixated brains. There is a striking similarity of MRI intensity profiles with photometric studies of the myeloarchitecture (Hopf, 1968a,b). In addition, theoretical studies (Hellwig, 1993) demonstrated the equivalence of Nissl-stained cytometric intensity profiles with Weigert-stained myelin profiles. A quantitative comparison of MRI intensity profiles with optical profiles of stained tissue is necessary.

The spatial resolution of the MRI data acquired here is $\approx 0.3 \mathrm{~mm}$, and thus at least one order of magnitude lower than any of the techniques mentioned before. However, optical profiles are typically smoothed in space (Schleicher and Zilles, 1990; Schleicher et al., 1998) in order to characterize gross features of layers. Results presented here suggest that perhaps a microscopic resolution is not required if a classification of cortical areas is sought for. However, at a higher resolution (say, $0.1 \mathrm{~mm}$ ), even more detail is revealed (Fatterpekar et al., 2002), leading to more powerful statistical classificators. It is ambitious but not impossible to implement scanning protocols with a spatial resolution of $0.1 \mathrm{~mm}$, at least if one focuses on a brain subregion (i.e. a lobe).

A critical issue of this study is to achieve a good signal-to-noise ratio (SNR) for acquiring high-resolution datasets of an acceptable quality. As for a given MR system, the MR signal strength is approximately proportional to the voxel volume, we simply scaled our standard scanning time of $20 \mathrm{~min}\left(1.5 \mathrm{~mm}^{3}\right.$ voxel volume) to $12 \mathrm{~h}$ ( $0.035 \mathrm{~mm}^{3}$ voxel volume). However, the SNR measured here was about two times better compared with our standard MRI datasets, which is probably due to the small substance load in the coil, and the fact that an isolated brain was scanned. One may consider to reduce the scanning time to $3 \mathrm{~h}$, accepting a SNR similar to those of standard MR datasets. We have rather chosen to conduct subsequent experiments with an isotropical resolution of $0.25 \mathrm{~mm}$.

It is an open issue how much the approach described here may be translated to in vivo studies. Our scanning time of $12 \mathrm{~h}$ is impossible to sustain for humans, and might even be hard to achieve when scanning anaethesized animals (e.g. monkeys). Nevertheless, progress in MR techniques (especially at ultra-high field strengths and/or using array receiver coils) will offer a significant reduction in scanning times, and let us expect to have such scanning techniques at our disposal within this decade. However, it is unclear at this time, how much unavoidable motion artefacts (e.g. from random head movements, swallowing, breathing and pulsation) influence the quality of highresolution scans. Note that in anatomical scans of a fixated brain, the tissue contrast is 'inverted': grey matter has a higher signal intensity than white matter. Modifying the procedure described here to analyze in vivo scans appears straightforward. Indeed, the pre-processing techniques were adapted from previous work in computing the cortical thickness (Kruggel and von Cramon, 2000), and involved mostly the change of parameter settings.

The possibility of studying the neocortical fine-structure by MR imaging, i.e. introducing a myeloarchitecture-related parcellation of an individual brain, offers exciting perspectives for the analysis of structure-function relationships in the brain on a mesoscopic level.

\section{References}

Amunts, K., Istomin, V., Schleicher, A., Zilles, K., 1995. Postnatal development of the human primary motor cortex: a quantitative cytoarchitectonic analysis. Anat. Embryol. 192, 557-571.

Amunts, K., Schmidt-Passos, F., Schleicher, A., Zilles, K., 1997. Postnatal development of interhemispheric asymmetry in the cytoarchitecture of human area 4. Anat. Embryol. 196, 393-402.

Amunts, K., Schleicher, A., Bürgel, U., Mohlberg, H., Uylings, H.B.M., Zilles, K., 1999. Broca's region revisited: cytoarchitecture and intersubject variability. J. Comp. Neurol. 412, 319-341.

Brodmann, K., 1908. Die vergleichende Lokalisationslehre der Großhirnrinde. Barth, Leipzig, English translation: Garey, L., 1992. Localisation in the cerebral cortex. Smith-Gordon, London.

Dale, A.M., Sereno, M.I., 1993. Improved localization of cortical activity by combining EEG and MEG with MRI cortical surface reconstruction: a linear approach. J. Cogn. Neurosci. 5, 162-176.

Eberly, D., 1999. Distance between point and triangle in 3D, http:/ /www.magic-software.com/graphics/src/dist/pt3tri3.pdf..

Fatterpekar, G.M., Naidich, T.P., Delman, B.N., Aguinaldo, J.G., Gultekin, S.H., Sherwood, C.C., Hof, P.R., Drayer, B.P., Fayad, Z.A., 2002. Cytoarchitecture of the human cerebral cortex: MR microscopy of excised specimens at 9.4 Tesla. Am. J. Neuroradiol. 23, 1313-1321.

Garland, M., Heckbert, P.S., 1999. Optimal triangulation and quadricbased surface simplification. J. Comp. Geom. 14, 49-65.

Geyer, S., Schleicher, A., Zilles, K., 1999. Areas 3a, 3b, and 1 of the human primary somatosensory cortex. Neuroimage 10, 63-83.

Hellwig, B., 1993. How the myelin picture of the human cerebral cortex can be computed from cytoarchitectonic data. A bridge between von Economo and Vogt. J. Hirnforsch. 34, 387-402.

Hopf, A., 1968a. Registration of the myeloarchitecture of the human frontal lobe with an extinction method. J. Hirnforsch. 10, 259-269.

Hopf, A., 1968b. Photometric studies on the myeloarchitecture of the human temporal lobe. J. Hirnforsch. 10, 285-297.

Kruggel, F., Lohmann, G., 1996. BRIAN (Brain Image Analysis): A toolkit for the analysis of multimodal brain data sets. In: Lemke, H.U., Inamura, K., Jaffe, C.C., Vannier, M.W. (Eds.), Computer Aided Radiology (CAR'96). Elsevier, Amsterdam, pp. 323-328.

Kruggel, F., von Cramon, D.Y., 2000. Measuring the neocortical thickness. In: Kimia, B. (Ed.), Workshop on Mathematical Models in Biomedical Image Analysis. IEEE Press, Los Alamitos, pp. 154-161.

Lee, J.H., Garwood, M., Menon, R., Adriany, G., Andersen, P., Truwit, C.L., Ugurbil, K., 1995. High contrast and fast three-dimensional magnetic resonance imaging at high fields. Magn. Reson. Med. 34, 308-312.

MacDonald, D., Kabani, N., Avis, D., Evans, A.C., 2000. Automated 3-D extraction of inner and outer surfaces of cerebral cortex from MRI. Neuroimage 12, 340-356. 
Payne, B.A., Toga, A.W., 1990. Surface mapping of brain function on 3D models. IEEE CGA 10, 33-41.

Pham, D.L., Prince, J.L., 1999. An adaptive fuzzy segmentation algorithm of magnetic resonance images. IEEE Trans. Med. Imag. 18, 737-752.

Rademacher, J., Caviness, V.S., Steinmetz, H., Galaburda, A.M., 1995. Topographical variation of the human primary cortices: implications for neuroimaging, brain mapping and neurobiology. Cereb. Cortex 3, 313-329.

Rajkowska, G., Goldman-Rakic, P.S., 1995a. Cytoarchitectonic definition of prefrontal areas in the normal human cortex: I. Remapping of areas 9 and 46 using quantitative criteria. Cereb. Cortex 5, 307-322.

Rajkowska, G., Goldman-Rakic, P.S., 1995b. Cytoarchitectonic definition of prefrontal areas in the normal human cortex: II. Variability in locations of areas 9 and 46 and relationship to the Talairach coordinate system. Cereb. Cortex 5, 323-337.

Sakissov, S.A., Filimonov, I.N., Kononowa, E.P., Preobraschenskaja, I.S., Kukuew, L.A., 1955. Atlas of the Cytoarchitectonics of the Human Cerebral Cortex. Medgiz, Moscow.

Schleicher, A., Zilles, K., 1990. A quantitative approach to cytoarchitectonics: analysis of structural inhomogeneities in nervous tissue using an image analyzer. J. Microsc. 157, 367-381.
Schleicher, A., Amunts, K., Geyer, S., Morosan, P., Zilles, K., 1998. Observer-independent method for microstructural parcellation of the cerebral cortex: a quantitative approach to cytoarchitectonics. Neuroimage 9, 165-177.

Thevenaz, P., Blu, T., Unser, M., 2000. Image interpolation and resampling. In: Handbook of Medical Image Processing. Academic Press, San Diego, pp. 393-420.

Vogt, C., Vogt, O., 1919. Allgemeinere Ergebnisse unserer Hirnforschung. J. Psych. Neurol. 25, 279-461.

von Economo, C., 1927. Zellaufbau der Großhirnrinde des Menschen. Springer, Wien, English translation: von Economo, C., Koskinas, G., 1929. The Cytoarchitectonics of the human cerebral cortex. Oxford University Press, Oxford.

Weis, S., Haug, H., Holoubek, B., Örün, H., 1989. The cerebral dominances: Quantitative morphology of the human cerebral cortex. Int. J. Neurosci. 47, 165-168.

Xu, C., Prince, J.L., 1998. Snakes, shapes, and gradient vector flow. IEEE Trans. Imag. Process. 1998, 359-369.

Zilles, K., Werners, R., Büsching, U., Schleicher, A., 1986. Ontogenesis of the laminar structure in areas 17 and 18 of the human visual cortex. Anat. Embryol. 174, 339-353. 